CRYSTALLOGRAPHIC COMMUNICATIONS

ISSN 2056-9890

Received 3 November 2021

Accepted 23 January 2022

Edited by J. Ellena, Universidade de Sâo Paulo, Brazil

Keywords: molecular structure; crystal structure; 5-amino-1 $\mathrm{H}$-benzimidazole-2 $(3 \mathrm{H})$-thione; hydrogen bond; Hirshfeld analysis; periodic calculations.

CCDC reference: 2143895

Supporting information: this article has supporting information at journals.iucr.org/e

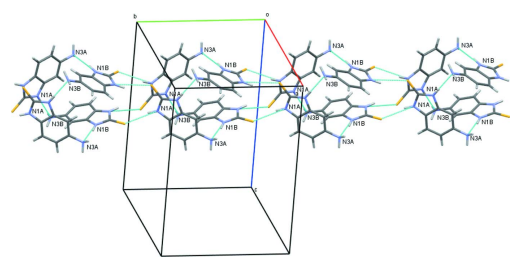

(c) (1) OPEN () ACCESS

\section{5-Amino-1H-benzimidazole-2(3H)-thione: molecular, crystal structure and Hirshfeld surface analysis}

\author{
Dilnoza Rakhmonova, ${ }^{\mathrm{a} *}$ Lobar Gapurova, ${ }^{\mathrm{a}}$ Surayyo Razzoqova, ${ }^{\mathrm{a}}$ Shakhnoza \\ Kadirova, ${ }^{a}$ Batirbay Torambetov, ${ }^{a} Z^{2}$ khra Kadirova ${ }^{a, b}$ and Svitlana Shishkina ${ }^{c}$
}

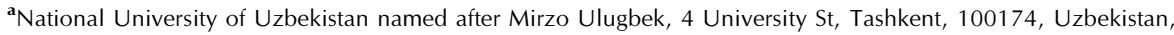
${ }^{\mathbf{b}}$ Uzbekistan-Japan Innovation Center of Youth, University Street 2B, 100095, Tashkent, Uzbekistan, and ${ }^{\mathbf{c}}$ State Scientific Institution "Institute for Single Crystals" of National Academy of Sciences of Ukraine, 60 Nauky ave., 61001 Kharkiv, Ukraine. *Correspondence e-mail: d.rakhmonova81@mail.ru

The title compound, $\mathrm{C}_{7} \mathrm{H}_{7} \mathrm{~N}_{3} \mathrm{~S}$, which has potential biological activity, can be used as a ligand in metal complexation. This compound exists as the thione tautomer in the crystal phase, which is confirmed by the study of its molecular structure. The amino group has pyramidal configuration. In the crystal phase, the two independent molecules in the asymmetric unit form tetramers as a result of $\mathrm{N}-\mathrm{H} \cdots \mathrm{S}$ hydrogen bonds. These tetramers are linked by $\mathrm{N}-\mathrm{H} \cdots \mathrm{N}$ hydrogen bonds, forming chains/tubes in the [010] direction. The Hirshfeld surface analysis showed that the highest contribution to the total surface is provided by $\mathrm{H} \cdots \mathrm{H}$ interactions as well as $\mathrm{S} \cdots \mathrm{H} / \mathrm{H} \cdots \mathrm{S}$ and $\mathrm{C} \cdots \mathrm{H} / \mathrm{H} \cdots \mathrm{C}$ contacts associated with $X-\mathrm{H} \cdots \mathrm{S}$ hydrogen bonds and $X-\mathrm{H} \cdots \mathrm{C}(\pi)$ interactions.

\section{Chemical context}

Benzimidazoles belong to an important class of heterocyclic compounds because of their wide spectra of biological activity. In particular, benzimidazole derivatives are known to possess antibacterial (Chkirate et al., 2020), antimicrobial (Alam et al., 2014), antitumor (Kharitonova et al., 2018; Galal et al., 2010), anti-inflammatory (Rathore et al., 2017), antioxidant (Anastassova et al., 2017), anthelmintic (Kenchappa et al., 2017), antifungal and cytotoxic (Leila et al., 2019) activity. They are also important as starting materials for terminal alkyne cyclotrimerization reactions (Xi et al., 2013) and are used as highly active catalysts for ethylene oligomerization (Haghverdi et al., 2018). The synthesis of 2-amino-1,3-benzimidazole-2-thione has been reported, prepared by first treating $o$-phenylenediamine $\mathrm{CS}_{2}$ in the presence of $\mathrm{KOH}$ under microwave irradiation to give the intermediate 1,3benzimidazole-2-thione. Nitration of the intermediate followed by reduction of the nitro group with iron powder and concentrated hydrochloric acid gave 2-amino-1,3-benzimidazole-2-thione in a moderately good yield (Samanta et al., 2013; Ahamed et al., 2013). Taking into account the possible biological activity of the obtained compound, it is important to study its molecular and crystal structures.

\section{Structural commentary}

Two independent molecules ( $A$ and $B$ ) comprise the asymmetric unit of the title compound (Fig. 1). The molecules 
slightly differ from each other in their degree of planarity: all non-hydrogen atoms lie in the same plane with an accuracy of $0.05 \AA$ in molecule $A$ and with an accuracy of $0.02 \AA$ in molecule $B$. Analysis of the molecular structure revealed that the $\mathrm{C}=\mathrm{S}$ tautomer is found in the crystal, as confirmed by the length of the $\mathrm{C} 7-\mathrm{S} 1$ bond [1.687 (3) $\AA$ in molecule $A$ and 1.684 (3) $\AA$ in molecule $B]$, the equal lengths of the $\mathrm{C} 7-\mathrm{N} 1$ and $\mathrm{C} 7-\mathrm{N} 2$ bonds $[1.345$ (3) and 1.347 (3) $\AA$ in molecule $A$ and 1.351 (3) and 1.349 (3) $\AA$ in molecule $B$ ] and the localization of hydrogen atoms at all the nitrogen atoms from the electron-density difference maps. The amino groups in both molecules are pyramidal, the sum of the bond angles centered at the nitrogen atom is $331.5^{\circ}$ in molecule $A$ and $340.9^{\circ}$ in molecule $B$.<smiles>Nc1ccc2[nH]c(=S)[nH]c2c1</smiles>

\section{Supramolecular features}

In the crystal, the molecules form tetramers as a result of the $\mathrm{N} 2 A-\mathrm{H} 2 N A \cdots \mathrm{S} 1 B$ and $\mathrm{N} 2 B-\mathrm{H} 2 N B \cdots \mathrm{S} 1 A$ hydrogen bonds (Fig. 2, Table 1). The tetramers are linked by $\mathrm{N} 1 A \cdots \mathrm{H} 1 N A-\mathrm{N} 3 B$ and $\mathrm{N} 1 B-\mathrm{H} 1 N B \cdots \mathrm{N} 3 A$ hydrogen bonds, forming a tube in the [010] direction (Figs. 3 and 4). Adjacent tubes are connected by weaker $\mathrm{N}-\mathrm{H} \cdots \mathrm{C}(\pi), \mathrm{C}-$ $\mathrm{H} \cdots \mathrm{S}, \mathrm{N}-\mathrm{H} \cdots \mathrm{S}$ and $\mathrm{C}-\mathrm{H} \cdots \mathrm{C}(\pi)$ interactions (Table 1 ).

\section{Hirshfeld surface analysis}

One of the modern methods for analysing intermolecular interactions is Hirshfeld surface analysis (Spackman \& Jaya-

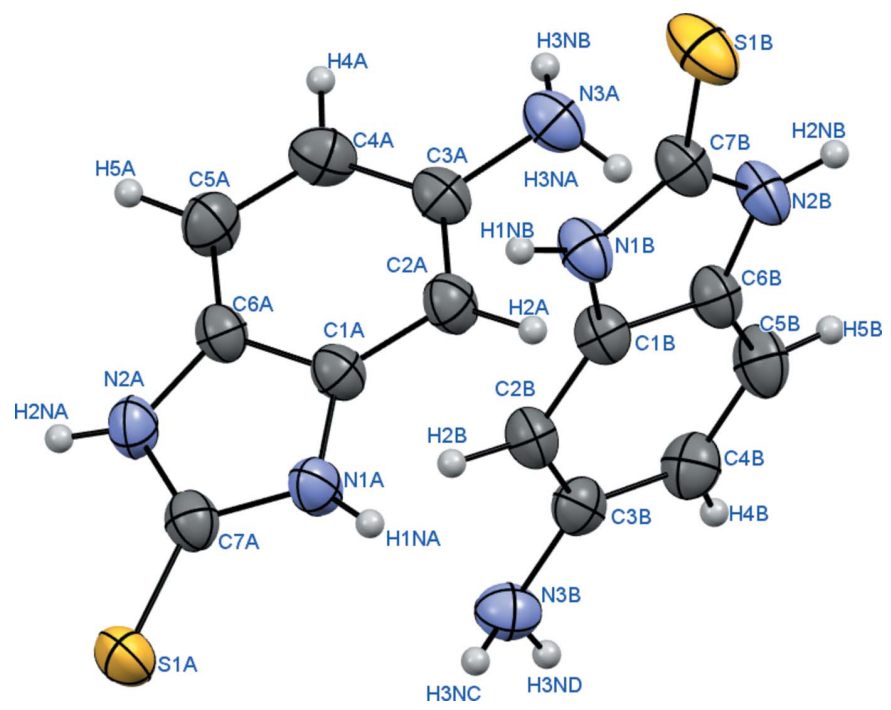

Figure 1

Molecular structures of molecules $A$ and $B$ showing the atom-labelling scheme. Displacement ellipsoids are drawn at the $50 \%$ probability level.
Table 1

Hydrogen-bond geometry $\left(\AA{ }^{\circ}\right)$.

\begin{tabular}{lllll}
\hline$D-\mathrm{H} \cdots A$ & $D-\mathrm{H}$ & $\mathrm{H} \cdots A$ & $D \cdots A$ & $D-\mathrm{H} \cdots A$ \\
\hline $\mathrm{N} 1 A-\mathrm{H} 1 N A \cdots \mathrm{N} 3 B$ & $0.80(3)$ & $2.06(3)$ & $2.856(3)$ & $176(3)$ \\
$\mathrm{N} 2 A-\mathrm{H} 2 N A \cdots \mathrm{S} 1 B^{\mathrm{i}}$ & $0.82(3)$ & $2.54(3)$ & $3.295(2)$ & $154(3)$ \\
$\mathrm{N} 3 A-\mathrm{H} 3 N A \cdots \mathrm{S} 1 A^{\text {ii }}$ & $0.84(4)$ & $2.75(4)$ & $3.551(3)$ & $159(3)$ \\
$\mathrm{N} 3 A-\mathrm{H} 3 N B \cdots \mathrm{C} 4 B^{\text {iii }}$ & $0.89(4)$ & $2.71(4)$ & $3.483(4)$ & $145(3)$ \\
$\mathrm{N} 3 A-\mathrm{H} 3 N B \cdots \mathrm{C} 5 B^{\text {iii }}$ & $0.89(4)$ & $2.81(4)$ & $3.643(4)$ & $155(3)$ \\
$\mathrm{N} 1 B-\mathrm{H} 1 N B \cdots \mathrm{N} 3 A^{\text {iv }}$ & $0.88(3)$ & $2.02(3)$ & $2.884(3)$ & $171(3)$ \\
$\mathrm{N} 2 B-\mathrm{H} 2 N B \cdots \mathrm{S} 1 A^{\text {v }}$ & $0.85(3)$ & $2.56(3)$ & $3.340(2)$ & $153(3)$ \\
$\mathrm{N} 3 B-\mathrm{H} 3 N C \cdots \mathrm{S} 1 B^{\text {vi }}$ & $0.86(3)$ & $2.91(3)$ & $3.672(3)$ & $149(2)$ \\
$\mathrm{N} 3 B-\mathrm{H} 3 N D \cdots \mathrm{S} 1 B^{\text {vii }}$ & $0.85(3)$ & $2.70(3)$ & $3.477(3)$ & $153(3)$ \\
$\mathrm{C} 5 A-\mathrm{H} 5 A \cdots \mathrm{S} 1 A^{\text {viii }}$ & $0.96(3)$ & $2.96(3)$ & $3.656(3)$ & $130.2(19)$ \\
$\mathrm{C} 5 B-\mathrm{H} 5 B \cdots \mathrm{C} 1 A^{\text {ii }}$ & $0.90(3)$ & $2.78(3)$ & $3.562(4)$ & $147(3)$ \\
\hline
\end{tabular}

Symmetry codes: (i) $\quad-x+1, y+1,-z+\frac{1}{2}$; $\quad$ (ii) $\quad-x+\frac{3}{2}, y-\frac{1}{2},-z+\frac{1}{2}$; $\quad$ (iii) $x,-y+1, z+\frac{1}{2}$; (iv) $-x+1, y,-z+\frac{1}{2}$; (v) $x, y-1, z$; (vi) $-x+1,-y+1,-z$; (vii) $x+\frac{1}{2}, y+\frac{1}{2}, z$; (viii) $x,-y+2, z+\frac{1}{2}$.

tilaka, 2009; Turner et al., 2017), which allows analysis of the interactions between molecules in a quantitative manner. The Hirshfeld surfaces of molecules $A$ and $B$ mapped over $d_{\text {norm }}$ proved to be very similar (Fig. 5). The red spots indicating strong interactions are found at both hydrogen atoms of the $\mathrm{NH}$ fragments as well as in the area of the nitrogen lone pair of the amino group. In addition, red spots are seen at the sulfur atom.

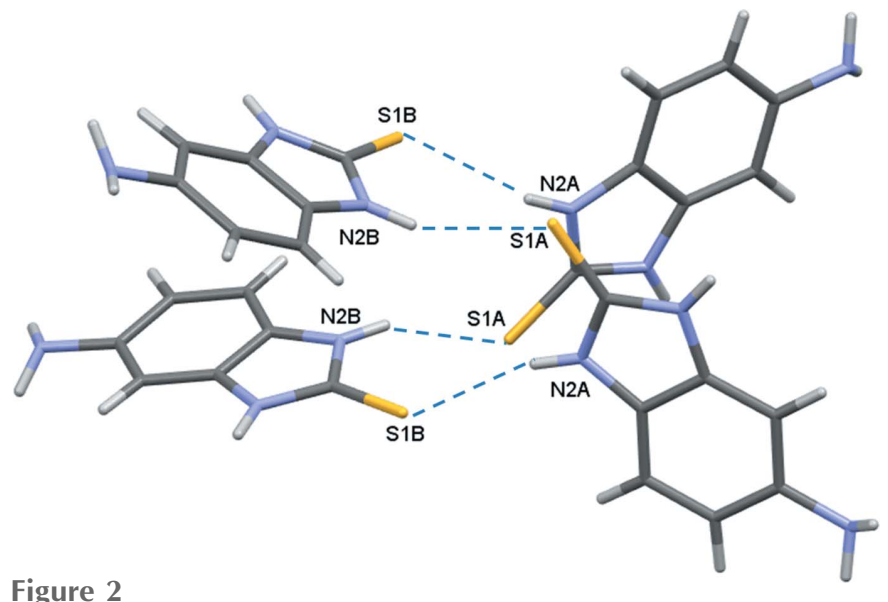

Tetramer of molecules $A$ and $B$ formed by $\mathrm{N} 2 A-\mathrm{H} 2 N A \cdots \mathrm{S} 1 B$ and $\mathrm{N} 2 B-\mathrm{H} 2 N B \cdots \mathrm{S} 1 A$ hydrogen bonds.

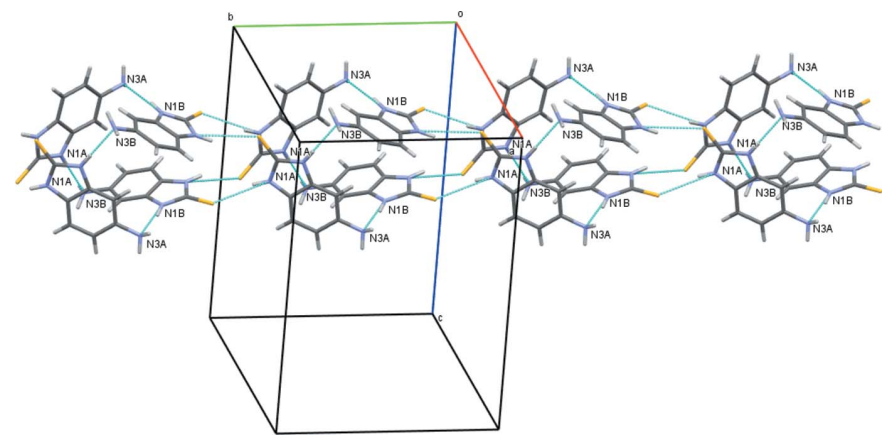

Figure 3

Chain/tube of tetramers linked by $\mathrm{N} 1 A \cdots \mathrm{H} 1 N A-\mathrm{N} 3 B$ and $\mathrm{N} 1 B-$ $\mathrm{H} 1 N B \cdots \mathrm{N} 3 A$ hydrogen bonds. 


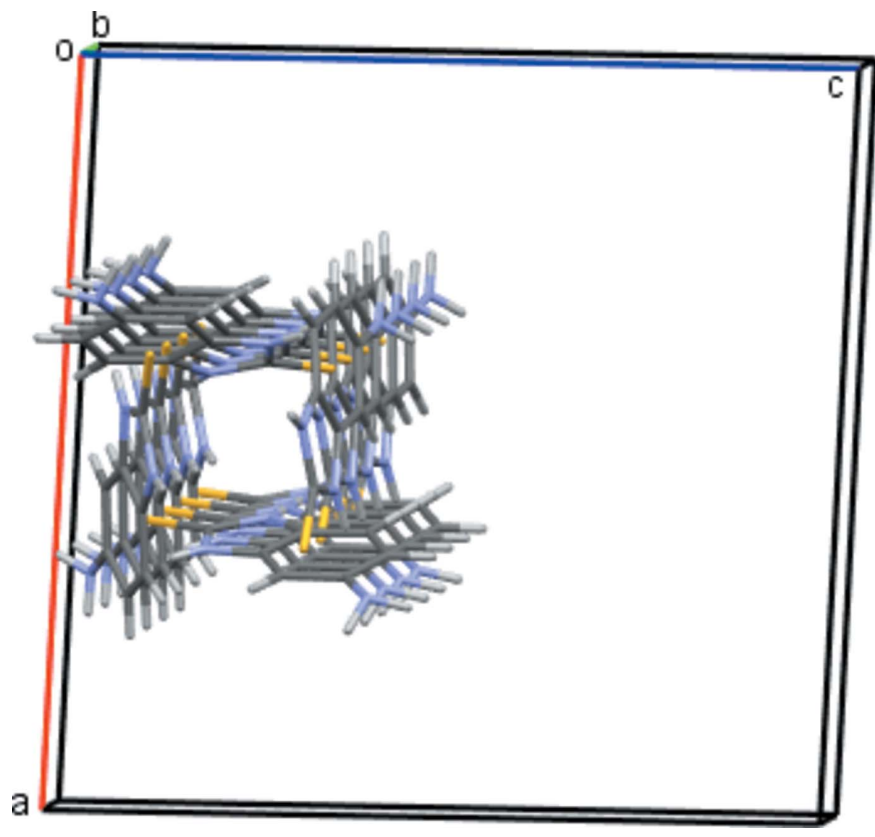

Figure 4

Projection of a tube in the $b$-axis direction.

Analysis of the fingerprint plots showed the presence of strong intermolecular interactions indicated as sharp spikes (Fig. $6 a, 6 b$ ). The most significant contribution to the total Hirshfeld surface is provided by $\mathrm{H} \cdots \mathrm{H}$ interactions in both molecules (Fig. $6 c, 6 g$ ). The contributions of S $\cdots \mathrm{H} / \mathrm{H} \cdots \mathrm{S}$ and C. . H/H $\cdots \mathrm{C}$ interactions associated with $X-\mathrm{H} \cdots \mathrm{S}$ and $X-$ $\mathrm{H} \cdots \mathrm{C}(\pi)$ hydrogen bonds are similar (Fig. $6 d-i$ ). Surprisingly, the contribution of $\mathrm{N} \cdots \mathrm{H} / \mathrm{H} \cdots \mathrm{N}$ interactions proved to be the lowest (Fig. $6 f, 6 j$ ). It may be explained by the participation of the nitrogen lone pair in hydrogen bonding as a proton acceptor.

\section{Database survey}

A search of the Cambridge Structural Database (Version 5.42, update of November 2020; Groom et al., 2016) revealed the structure of the monohydrate of the title compound (ODAXID; Hadjikakou \& Light, 2016). It should be noted that the amino group was refined as planar in this structure. However, analysis of the intermolecular interactions showed that this amino group participates in a hydrogen bond with the hydrate water molecule as a proton acceptor. Such a hydrogen

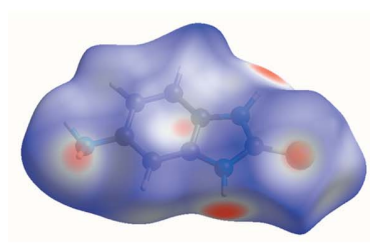

Molecule A

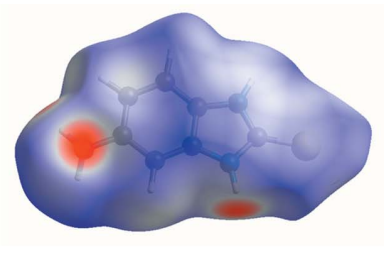

Molecule B
Figure 5

Hirshfeld surfaces mapped over $d_{\text {norm }}$ calculated for molecules $A$ and $B$.
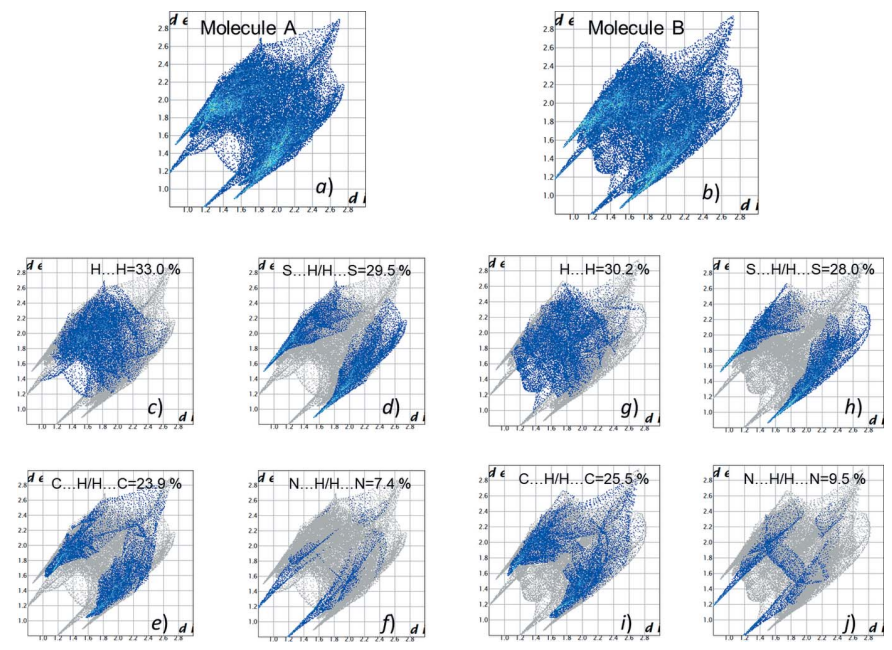

Figure 6

Two-dimensional Hirshfeld fingerprint plot of all contacts for molecules $A(a)$ and $B(b)$ and those delineated into $\mathrm{H} \cdots \mathrm{H}(c, g), \mathrm{S} \cdots \mathrm{H} / \mathrm{H} \cdots \mathrm{S}(d$, $h), \mathrm{C} \cdots \mathrm{H} / \mathrm{H} \cdots \mathrm{C}(e, i)$ and $\mathrm{N} \cdots \mathrm{H} / \mathrm{H} \cdots \mathrm{N}(f, j)$ contacts.

bonding has to result in pyramidalization of the amino group. To check this presumption, we have optimized the ODAXID structure with a periodic boundary using the PBE functional (Adamo \& Barone, 1999) within Quantum Espresso (Giannozzi et al., 2009, 2017). The unit-cell parameters were fixed while the molecular structures of both molecules found in the asymmetric unit were optimized. The result of this optimization shows that the amino group has to be pyramidal (Fig. 7).

\section{Crystallization}

5-Amino- $1 H$-benzimidazole-2(3H)-thione was purchased from Sigma-Aldrich for use as a ligand in complexation with metals. The reaction of the title compound with nickel acetate in an aqueous alcoholic medium did not result in complex formation. The formed colourless needle-like crystals proved to be anhydrous form of the ligand with $T_{\text {melt. }}=513-517 \mathrm{~K}$.

\section{Refinement}

Crystal data, data collection and structure refinement details are summarized in Table 2. All the hydrogen atoms were located in difference-Fourier maps and refined using an isotropic approximation.
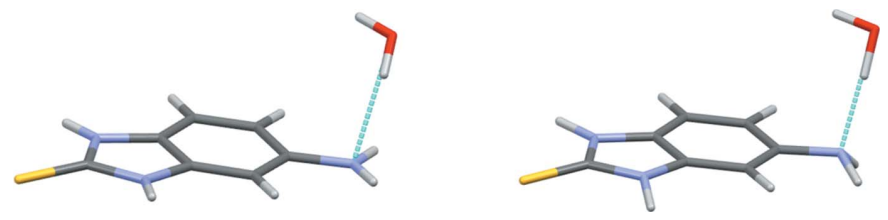

Figure 7

Configuration of the amino group in the structure of ODAXID calculated from the experimental data (Hadjikakou \& Light, 2016) and obtained after optimization with a periodic boundary. 
Table 2

Experimental details.

\begin{tabular}{|c|c|}
\hline \multicolumn{2}{|l|}{ Crystal data } \\
\hline Chemical formula & $\mathrm{C}_{7} \mathrm{H}_{7} \mathrm{~N}_{3} \mathrm{~S}$ \\
\hline$M_{\mathrm{r}}$ & 165.22 \\
\hline Crystal system, space group & Monoclinic, $C 2 / c$ \\
\hline Temperature (K) & 293 \\
\hline$a, b, c(\AA)$ & $\begin{array}{l}16.1179(14), 11.8796(11) \\
\quad 16.5649(15)\end{array}$ \\
\hline$\beta\left(^{\circ}\right)$ & $91.974(8)$ \\
\hline$V\left(\AA^{3}\right)$ & $3169.9(5)$ \\
\hline$Z$ & 16 \\
\hline Radiation type & Мо $K \alpha$ \\
\hline$\mu\left(\mathrm{mm}^{-1}\right)$ & 0.34 \\
\hline Crystal size (mm) & $0.80 \times 0.26 \times 0.08$ \\
\hline \multicolumn{2}{|l|}{ Data collection } \\
\hline Diffractometer & Xcalibur, Sapphire3 \\
\hline Absorption correction & $\begin{array}{l}\text { Multi-scan (CrysAlis PRO; Rigaku } \\
\text { OD, 2018) }\end{array}$ \\
\hline$T_{\min }, T_{\max }$ & $0.370,1.000$ \\
\hline $\begin{array}{l}\text { No. of measured, independent and } \\
\text { observed }[I>2 \sigma(I)] \text { reflections }\end{array}$ & $12390,2787,2417$ \\
\hline$R_{\text {int }}$ & 0.079 \\
\hline$(\sin \theta / \lambda)_{\max }\left(\AA^{-1}\right)$ & 0.595 \\
\hline \multicolumn{2}{|l|}{ Refinement } \\
\hline$R\left[F^{2}>2 \sigma\left(F^{2}\right)\right], w R\left(F^{2}\right), S$ & $0.052,0.138,1.05$ \\
\hline No. of reflections & 2787 \\
\hline No. of parameters & 255 \\
\hline $\mathrm{H}$-atom treatment & All H-atom parameters refined \\
\hline$\Delta \rho_{\max }, \Delta \rho_{\min }\left(\mathrm{e} \AA^{-3}\right)$ & $0.33,-0.27$ \\
\hline
\end{tabular}

Computer programs: CrysAlis PRO (Rigaku OD, 2018), SHELXT2014/5 (Sheldrick, 2015a), SHELXL2016/6 (Sheldrick, 2015b), Mercury (Macrae et al., 2020) and Olex2 (Dolomanov et al., 2009).

\section{References}

Adamo, C. \& Barone, V. (1999). J. Chem. Phys. 110, 6158-6170.

Ahamed, M. R., Narren, S. F. \& Sadiq, A. S. (2013). J. Al-Nahrain Uni. 16, 77-83.

Alam, F., Dey, B. K., Sharma, K., Chakraborty, A. \& Kalita, P. (2014). Int. J. Drug Res. Tech. 4(3), 31-38.

Anastassova, N., Mavrova, A., Yancheva, D., Kondeva-Burdina, M., Tzankova, V., Stoyanov, S., Shivachev, B. L. \& Nikolova, R. P. (2017). Arab. J. Chem. 11, 353-369.

Chkirate, K., Karrouchi, K., Dege, N., Sebbar, N. K., Ejjoummany, A., Radi, S., Adarsh, N. N., Talbaoui, A., Ferbinteanu, M., Essassi, E. M. \& Garcia, Y. (2020). New J. Chem. 44, 2210-2221.

Dolomanov, O. V., Bourhis, L. J., Gildea, R. J., Howard, J. A. K. \& Puschmann, H. (2009). J. Appl. Cryst. 42, 339-341.
Galal, S. A., Hegab, K. H., Hashem, A. M. \& Youssef, N. S. (2010). Eur. J. Med. Chem. 45, 5685-5691.

Giannozzi, P., Andreussi, O., Brumme, T., Bunau, O., Buongiorno Nardelli, M., Calandra, M., Car, R., Cavazzoni, C., Ceresoli, D., Cococcioni, M., Colonna, N., Carnimeo, I., Dal Corso, A., de Gironcoli, S., Delugas, P., DiStasio, R. A., Ferretti, A., Floris, A., Fratesi, G., Fugallo, G., Gebauer, R., Gerstmann, U., Giustino, F., Gorni, T., Jia, J., Kawamura, M., Ko, H. Y., Kokalj, A., Küçükbenli, E., Lazzeri, M., Marsili, M., Marzari, N., Mauri, F., Nguyen, N. L., Nguyen, H. V., Otero-de-la-Roza, A., Paulatto, L., Poncé, S., Rocca, D., Sabatini, R., Santra, B., Schlipf, M., Seitsonen, A. P., Smogunov, A., Timrov, I., Thonhauser, T., Umari, P., Vast, N., Wu, X. \& Baroni, S. (2017). J. Phys. Condens. Matter, 29, 465901.

Giannozzi, P., Baroni, S., Bonini, N., Calandra, M., Car, R., Cavazzoni, C., Ceresoli, D., Chiarotti, G. L., Cococcioni, M., Dabo, I., Dal Corso, A., de Gironcoli, S., Fabris, S., Fratesi, G., Gebauer, R., Gerstmann, U., Gougoussis, C., Kokalj, A., Lazzeri, M., MartinSamos, L., Marzari, N., Mauri, F., Mazzarello, R., Paolini, S., Pasquarello, A., Paulatto, L., Sbraccia, C., Scandolo, S., Sclauzero, G., Seitsonen, A. P., Smogunov, A., Umari, P. \& Wentzcovitch, R. M. (2009). J. Phys. Condens. Matter, 21, 395502.

Groom, C. R., Bruno, I. J., Lightfoot, M. P. \& Ward, S. C. (2016). Acta Cryst. B72, 171-179.

Hadjikakou, S. \& Light, M. E. (2016). Private communication (refcode ODAXID). CCDC, Cambridge, England.

Haghverdi, M., Tadjarodi, A., Bahri-Laleh, N. \& NekoomaneshHaghighi, M. (2018). Appl. Organomet. Chem. 32, e4015.

Kenchappa, R., Bodke, Y. D., Telkar, S. \& Aruna Sindhe, M. (2017). J. Chem. Biol. 10, 11-23.

Kharitonova, M. I., Konstantinova, I. D. \& Miroshnikov, A. I. (2018). Russ. Chem. Rev. 87, 1111-1138.

Leila, Z., Zeinab, F., Kamiar, Z., Fatemeh Bi Bi, M., Asghar, J. \& Soghra, K. (2019). Res. Pharma. Sci. 14, 504-514.

Macrae, C. F., Sovago, I., Cottrell, S. J., Galek, P. T. A., McCabe, P., Pidcock, E., Platings, M., Shields, G. P., Stevens, J. S., Towler, M. \& Wood, P. A. (2020). J. Appl. Cryst. 53, 226-235.

Rathore, A., Sudhakar, R., Ahsan, M. J., Ali, A., Subbarao, N., Jadav, S. S., Umar, S. \& Yar, M. S. (2017). Bioorg. Chem. 70, 107-117.

Rigaku OD (2018). CrysAlis PRO. Rigaku Oxford Diffraction, Yarnton, England.

Samanta, S., Lim, T. L. \& Lam, Y. (2013). ChemMedChem, 8, 994 1001.

Sheldrick, G. M. (2015a). Acta Cryst. A71, 3-8.

Sheldrick, G. M. (2015b). Acta Cryst. A71, 3-8.

Spackman, M. A. \& Jayatilaka, D. (2009). CrystEngComm, 11, 19-32.

Turner, M. J., McKinnon, J. J., Wolff, S. K., Grimwood, D. J., Spackman, P. R., Jayatilaka, D. \& Spackman, M. A. (2017). CrystalExplorer17. University of Western Australia. http://Hirshfeldsurface.net

Xi, C., Sun, Z. \& Liu, Y. (2013). Dalton Trans. 42, 13327-13330. 


\section{supporting information}

Acta Cryst. (2022). E78, 231-234 [https://doi.org/10.1107/S2056989022000792]

\section{5-Amino-1H-benzimidazole-2(3H)-thione: molecular, crystal structure and Hirshfeld surface analysis}

\section{Dilnoza Rakhmonova, Lobar Gapurova, Surayyo Razzoqova, Shakhnoza Kadirova, Batirbay Torambetov, Zukhra Kadirova and Svitlana Shishkina}

\section{Computing details}

Data collection: CrysAlis PRO (Rigaku OD, 2018); cell refinement: CrysAlis PRO (Rigaku OD, 2018); data reduction: CrysAlis PRO (Rigaku OD, 2018); program(s) used to solve structure: SHELXT2014/5 (Sheldrick, 2015a); program(s) used to refine structure: SHELXL2016/6 (Sheldrick, 2015b); molecular graphics: Mercury (Macrae et al., 2020); software used to prepare material for publication: Olex2 (Dolomanov et al., 2009).

5-Amino-1H-benzimidazole-2(3H)-thione

Crystal data

$\mathrm{C}_{7} \mathrm{H}_{7} \mathrm{~N}_{3} \mathrm{~S}$

$M_{r}=165.22$

Monoclinic, $C 2 / c$

$a=16.1179(14) \AA$

$b=11.8796(11) \AA$

$c=16.5649(15) \AA$

$\beta=91.974(8)^{\circ}$

$V=3169.9(5) \AA^{3}$

$Z=16$

\section{Data collection}

Xcalibur, Sapphire3 diffractometer

Radiation source: Enhance (Mo) X-ray Source Detector resolution: 16.1827 pixels $\mathrm{mm}^{-1}$ $\omega$ scans

Absorption correction: multi-scan

(CrysAlisPro; Rigaku OD, 2018)

$T_{\min }=0.370, T_{\max }=1.000$

\section{Refinement}

Refinement on $F^{2}$

Least-squares matrix: full

$R\left[F^{2}>2 \sigma\left(F^{2}\right)\right]=0.052$

$w R\left(F^{2}\right)=0.138$

$S=1.05$

2787 reflections

255 parameters

0 restraints
$F(000)=1376$

$D_{\mathrm{x}}=1.385 \mathrm{Mg} \mathrm{m}^{-3}$

Mo $K \alpha$ radiation, $\lambda=0.71073 \AA$

Cell parameters from 2937 reflections

$\theta=3.5-26.9^{\circ}$

$\mu=0.34 \mathrm{~mm}^{-1}$

$T=293 \mathrm{~K}$

Plate, colorless

$0.80 \times 0.26 \times 0.08 \mathrm{~mm}$

12390 measured reflections

2787 independent reflections

2417 reflections with $I>2 \sigma(I)$

$R_{\text {int }}=0.079$

$\theta_{\max }=25.0^{\circ}, \theta_{\min }=3.2^{\circ}$

$h=-19 \rightarrow 18$

$k=-14 \rightarrow 14$

$l=-19 \rightarrow 19$

Hydrogen site location: difference Fourier map

All $\mathrm{H}$-atom parameters refined

$w=1 /\left[\sigma^{2}\left(F_{\mathrm{o}}^{2}\right)+(0.0719 P)^{2}+1.8442 P\right]$

where $P=\left(F_{\mathrm{o}}^{2}+2 F_{\mathrm{c}}^{2}\right) / 3$

$(\Delta / \sigma)_{\max }=0.001$

$\Delta \rho_{\max }=0.33$ e $\AA^{-3}$

$\Delta \rho_{\min }=-0.27$ e $\AA^{-3}$ 


\section{Special details}

Geometry. All esds (except the esd in the dihedral angle between two 1.s. planes) are estimated using the full covariance matrix. The cell esds are taken into account individually in the estimation of esds in distances, angles and torsion angles; correlations between esds in cell parameters are only used when they are defined by crystal symmetry. An approximate (isotropic) treatment of cell esds is used for estimating esds involving l.s. planes.

Fractional atomic coordinates and isotropic or equivalent isotropic displacement parameters $\left(\AA^{2}\right)$

\begin{tabular}{|c|c|c|c|c|}
\hline & $x$ & $y$ & $z$ & $U_{\text {iso }} * / U_{\text {eq }}$ \\
\hline S1B & $0.37352(5)$ & $0.24474(6)$ & $0.14471(5)$ & $0.0546(3)$ \\
\hline N1B & $0.44866(14)$ & $0.44339(17)$ & $0.11231(14)$ & $0.0388(5)$ \\
\hline H1NB & $0.402(2)$ & $0.477(3)$ & $0.098(2)$ & $0.060(9)^{*}$ \\
\hline $\mathrm{N} 2 \mathrm{~B}$ & $0.53394(13)$ & $0.31429(19)$ & $0.15625(14)$ & $0.0406(5)$ \\
\hline $\mathrm{H} 2 \mathrm{NB}$ & $0.5498(18)$ & $0.248(2)$ & $0.1694(18)$ & $0.048(8)^{*}$ \\
\hline N3B & $0.66860(17)$ & $0.7248(2)$ & $0.07344(15)$ & $0.0425(6)$ \\
\hline $\mathrm{H} 3 \mathrm{NC}$ & $0.6461(19)$ & $0.754(2)$ & $0.031(2)$ & $0.047(9)^{*}$ \\
\hline H3ND & $0.721(2)$ & $0.729(2)$ & $0.0726(18)$ & $0.049(9)^{*}$ \\
\hline $\mathrm{C} 1 \mathrm{~B}$ & $0.52713(14)$ & 0.4917 (2) & 0.11265 (14) & $0.0345(6)$ \\
\hline $\mathrm{C} 2 \mathrm{~B}$ & $0.55387(16)$ & 0.5967 (2) & $0.08966(15)$ & $0.0365(6)$ \\
\hline $\mathrm{H} 2 \mathrm{~B}$ & $0.5159(17)$ & $0.655(2)$ & $0.0704(16)$ & $0.049(8)^{*}$ \\
\hline C3B & $0.63884(15)$ & $0.6175(2)$ & 0.09525 (14) & $0.0360(6)$ \\
\hline C4B & $0.69335(17)$ & $0.5355(2)$ & $0.12677(17)$ & $0.0428(6)$ \\
\hline H4B & $0.748(2)$ & $0.556(2)$ & $0.1314(18)$ & $0.051(8)^{*}$ \\
\hline C5B & $0.66596(17)$ & $0.4308(2)$ & $0.15038(18)$ & $0.0449(7)$ \\
\hline H5B & $0.699(2)$ & $0.378(3)$ & $0.174(2)$ & $0.063(9)^{*}$ \\
\hline C6B & $0.58190(15)$ & $0.4093(2)$ & $0.14187(15)$ & $0.0365(6)$ \\
\hline C7B & $0.45329(16)$ & $0.3353(2)$ & $0.13759(15)$ & $0.0382(6)$ \\
\hline S1A & $0.60205(5)$ & $1.05022(6)$ & $0.13822(4)$ & $0.0479(3)$ \\
\hline N1A & $0.63692(14)$ & $0.85370(18)$ & $0.21461(13)$ & $0.0397(5)$ \\
\hline H1NA & $0.6481(17)$ & $0.819(3)$ & $0.1755(18)$ & $0.044(8)^{*}$ \\
\hline $\mathrm{N} 2 \mathrm{~A}$ & $0.59495(13)$ & $0.9834(2)$ & $0.29505(13)$ & $0.0383(5)$ \\
\hline $\mathrm{H} 2 \mathrm{NA}$ & $0.5855(18)$ & $1.049(3)$ & $0.3087(18)$ & $0.046(8)^{*}$ \\
\hline N3A & $0.69384(16)$ & $0.57892(19)$ & $0.43321(16)$ & $0.0420(6)$ \\
\hline H3NA & $0.737(2)$ & $0.556(3)$ & $0.411(2)$ & $0.061(11)^{*}$ \\
\hline $\mathrm{H} 3 \mathrm{NB}$ & $0.705(2)$ & $0.580(3)$ & $0.486(2)$ & $0.067(10)^{*}$ \\
\hline $\mathrm{C} 1 \mathrm{~A}$ & $0.63986(15)$ & $0.8070(2)$ & 0.29109 (14) & $0.0343(6)$ \\
\hline $\mathrm{C} 2 \mathrm{~A}$ & $0.66733(16)$ & $0.7028(2)$ & $0.31939(16)$ & $0.0382(6)$ \\
\hline $\mathrm{H} 2 \mathrm{~A}$ & $0.6856(16)$ & $0.644(2)$ & $0.2830(17)$ & $0.044(7)^{*}$ \\
\hline $\mathrm{C} 3 \mathrm{~A}$ & $0.66781(15)$ & $0.6855(2)$ & $0.40200(15)$ & $0.0354(6)$ \\
\hline $\mathrm{C} 4 \mathrm{~A}$ & $0.63934(17)$ & $0.7692(2)$ & $0.45370(17)$ & $0.0421(6)$ \\
\hline $\mathrm{H} 4 \mathrm{~A}$ & $0.6398(18)$ & $0.753(2)$ & $0.5104(19)$ & $0.050(8)^{*}$ \\
\hline C5A & $0.61082(18)$ & $0.8722(2)$ & $0.42509(16)$ & $0.0432(6)$ \\
\hline H5A & $0.5872(16)$ & $0.927(2)$ & $0.4604(16)$ & $0.039(7)^{*}$ \\
\hline C6A & $0.61262(15)$ & $0.8897(2)$ & $0.34291(15)$ & $0.0344(5)$ \\
\hline C7A & $0.61113(15)$ & $0.9613(2)$ & $0.21741(15)$ & $0.0373(6)$ \\
\hline
\end{tabular}


Atomic displacement parameters $\left(\AA^{2}\right)$

\begin{tabular}{lllllll}
\hline & $U^{11}$ & $U^{22}$ & $U^{33}$ & $U^{12}$ & $U^{13}$ & $U^{23}$ \\
\hline S1B & $0.0502(5)$ & $0.0400(4)$ & $0.0723(6)$ & $-0.0110(3)$ & $-0.0143(4)$ & $0.0145(3)$ \\
N1B & $0.0347(12)$ & $0.0318(11)$ & $0.0493(13)$ & $0.0011(9)$ & $-0.0089(10)$ & $0.0041(10)$ \\
N2B & $0.0421(12)$ & $0.0278(12)$ & $0.0514(13)$ & $0.0040(9)$ & $-0.0072(10)$ & $0.0060(10)$ \\
N3B & $0.0432(14)$ & $0.0435(14)$ & $0.0408(13)$ & $-0.0074(11)$ & $0.0015(11)$ & $0.0010(11)$ \\
C1B & $0.0343(13)$ & $0.0337(13)$ & $0.0350(12)$ & $0.0000(10)$ & $-0.0058(10)$ & $-0.0007(10)$ \\
C2B & $0.0388(14)$ & $0.0304(13)$ & $0.0399(13)$ & $0.0032(11)$ & $-0.0035(11)$ & $0.0005(11)$ \\
C3B & $0.0421(14)$ & $0.0340(13)$ & $0.0320(12)$ & $-0.0014(11)$ & $0.0004(11)$ & $-0.0065(10)$ \\
C4B & $0.0343(14)$ & $0.0460(16)$ & $0.0477(15)$ & $0.0009(12)$ & $-0.0031(12)$ & $-0.0043(12)$ \\
C5B & $0.0383(15)$ & $0.0410(15)$ & $0.0546(16)$ & $0.0082(12)$ & $-0.0109(13)$ & $0.0001(13)$ \\
C6B & $0.0379(13)$ & $0.0321(13)$ & $0.0391(13)$ & $0.0036(11)$ & $-0.0056(11)$ & $0.0005(11)$ \\
C7B & $0.0439(14)$ & $0.0322(13)$ & $0.0379(13)$ & $-0.0004(11)$ & $-0.0069(11)$ & $0.0027(11)$ \\
S1A & $0.0595(5)$ & $0.0385(4)$ & $0.0459(4)$ & $0.0055(3)$ & $0.0033(3)$ & $0.0090(3)$ \\
N1A & $0.0565(14)$ & $0.0307(12)$ & $0.0322(11)$ & $0.0023(10)$ & $0.0034(10)$ & $-0.0023(10)$ \\
N2A & $0.0462(13)$ & $0.0302(12)$ & $0.0384(12)$ & $0.0063(10)$ & $0.0006(10)$ & $-0.0047(10)$ \\
N3A & $0.0443(14)$ & $0.0378(13)$ & $0.0432(13)$ & $-0.0024(10)$ & $-0.0074(12)$ & $0.0044(11)$ \\
C1A & $0.0376(13)$ & $0.0309(13)$ & $0.0342(12)$ & $-0.0024(10)$ & $-0.0023(10)$ & $-0.0008(10)$ \\
C2A & $0.0455(15)$ & $0.0297(13)$ & $0.0392(14)$ & $-0.0013(11)$ & $-0.0011(11)$ & $-0.0038(11)$ \\
C3A & $0.0358(13)$ & $0.0318(13)$ & $0.0383(13)$ & $-0.0060(10)$ & $-0.0054(10)$ & $0.0000(11)$ \\
C4A & $0.0474(15)$ & $0.0447(15)$ & $0.0338(14)$ & $-0.0055(12)$ & $-0.0048(12)$ & $0.0008(12)$ \\
C5A & $0.0542(16)$ & $0.0397(15)$ & $0.0354(14)$ & $0.0016(12)$ & $-0.0008(12)$ & $-0.0068(12)$ \\
C6A & $0.0358(13)$ & $0.0305(13)$ & $0.0368(13)$ & $0.0002(10)$ & $-0.0024(10)$ & $-0.0014(10)$ \\
C7A & $0.0355(13)$ & $0.0330(13)$ & $0.0430(15)$ & $-0.0003(10)$ & $-0.0017(11)$ & $-0.0011(11)$ \\
& & & & & & \\
& & & & & & \\
& & & & &
\end{tabular}

Geometric parameters $\left(\AA,{ }^{o}\right)$

\begin{tabular}{|c|c|c|c|}
\hline $\mathrm{S} 1 \mathrm{~B}-\mathrm{C} 7 \mathrm{~B}$ & $1.684(3)$ & $\mathrm{S} 1 \mathrm{~A}-\mathrm{C} 7 \mathrm{~A}$ & $1.686(3)$ \\
\hline $\mathrm{N} 1 \mathrm{~B}-\mathrm{C} 7 \mathrm{~B}$ & $1.351(3)$ & $\mathrm{N} 1 \mathrm{~A}-\mathrm{C} 7 \mathrm{~A}$ & $1.346(3)$ \\
\hline $\mathrm{N} 1 \mathrm{~B}-\mathrm{C} 1 \mathrm{~B}$ & $1.389(3)$ & $\mathrm{N} 1 \mathrm{~A}-\mathrm{C} 1 \mathrm{~A}$ & $1.382(3)$ \\
\hline $\mathrm{N} 1 \mathrm{~B}-\mathrm{H} 1 \mathrm{NB}$ & $0.88(3)$ & $\mathrm{N} 1 \mathrm{~A}-\mathrm{H} 1 \mathrm{NA}$ & $0.80(3)$ \\
\hline $\mathrm{N} 2 \mathrm{~B}-\mathrm{C} 7 \mathrm{~B}$ & $1.349(3)$ & $\mathrm{N} 2 \mathrm{~A}-\mathrm{C} 7 \mathrm{~A}$ & $1.347(3)$ \\
\hline $\mathrm{N} 2 \mathrm{~B}-\mathrm{C} 6 \mathrm{~B}$ & $1.393(3)$ & $\mathrm{N} 2 \mathrm{~A}-\mathrm{C} 6 \mathrm{~A}$ & $1.390(3)$ \\
\hline $\mathrm{N} 2 \mathrm{~B}-\mathrm{H} 2 \mathrm{NB}$ & $0.85(3)$ & $\mathrm{N} 2 \mathrm{~A}-\mathrm{H} 2 \mathrm{NA}$ & $0.82(3)$ \\
\hline $\mathrm{N} 3 \mathrm{~B}-\mathrm{C} 3 \mathrm{~B}$ & $1.413(3)$ & $\mathrm{N} 3 \mathrm{~A}-\mathrm{C} 3 \mathrm{~A}$ & $1.426(3)$ \\
\hline $\mathrm{N} 3 \mathrm{~B}-\mathrm{H} 3 \mathrm{NC}$ & $0.86(3)$ & $\mathrm{N} 3 \mathrm{~A}-\mathrm{H} 3 \mathrm{NA}$ & $0.84(4)$ \\
\hline $\mathrm{N} 3 \mathrm{~B}-\mathrm{H} 3 \mathrm{ND}$ & $0.85(3)$ & $\mathrm{N} 3 \mathrm{~A}-\mathrm{H} 3 \mathrm{NB}$ & $0.89(4)$ \\
\hline $\mathrm{C} 1 \mathrm{~B}-\mathrm{C} 2 \mathrm{~B}$ & $1.378(4)$ & $\mathrm{C} 1 \mathrm{~A}-\mathrm{C} 6 \mathrm{~A}$ & $1.386(3)$ \\
\hline $\mathrm{C} 1 \mathrm{~B}-\mathrm{C} 6 \mathrm{~B}$ & $1.394(3)$ & $\mathrm{C} 1 \mathrm{~A}-\mathrm{C} 2 \mathrm{~A}$ & $1.390(4)$ \\
\hline $\mathrm{C} 2 \mathrm{~B}-\mathrm{C} 3 \mathrm{~B}$ & $1.391(4)$ & $\mathrm{C} 2 \mathrm{~A}-\mathrm{C} 3 \mathrm{~A}$ & $1.384(3)$ \\
\hline $\mathrm{C} 2 \mathrm{~B}-\mathrm{H} 2 \mathrm{~B}$ & $0.97(3)$ & $\mathrm{C} 2 \mathrm{~A}-\mathrm{H} 2 \mathrm{~A}$ & $0.98(3)$ \\
\hline $\mathrm{C} 3 \mathrm{~B}-\mathrm{C} 4 \mathrm{~B}$ & $1.401(4)$ & $\mathrm{C} 3 \mathrm{~A}-\mathrm{C} 4 \mathrm{~A}$ & $1.400(4)$ \\
\hline $\mathrm{C} 4 \mathrm{~B}-\mathrm{C} 5 \mathrm{~B}$ & $1.380(4)$ & $\mathrm{C} 4 \mathrm{~A}-\mathrm{C} 5 \mathrm{~A}$ & $1.385(4)$ \\
\hline $\mathrm{C} 4 \mathrm{~B}-\mathrm{H} 4 \mathrm{~B}$ & $0.92(3)$ & $\mathrm{C} 4 \mathrm{~A}-\mathrm{H} 4 \mathrm{~A}$ & $0.96(3)$ \\
\hline $\mathrm{C} 5 \mathrm{~B}-\mathrm{C} 6 \mathrm{~B}$ & $1.381(4)$ & $\mathrm{C} 5 \mathrm{~A}-\mathrm{C} 6 \mathrm{~A}$ & $1.378(4)$ \\
\hline $\mathrm{C} 5 \mathrm{~B}-\mathrm{H} 5 \mathrm{~B}$ & $0.90(3)$ & $\mathrm{C} 5 \mathrm{~A}-\mathrm{H} 5 \mathrm{~A}$ & $0.96(3)$ \\
\hline
\end{tabular}




\begin{tabular}{|c|c|}
\hline $\mathrm{C} 7 \mathrm{~B}-\mathrm{N} 1 \mathrm{~B}-\mathrm{C} 1 \mathrm{~B}$ & $110.5(2)$ \\
\hline $\mathrm{C} 7 \mathrm{~B}-\mathrm{N} 1 \mathrm{~B}-\mathrm{H} 1 \mathrm{NB}$ & $124(2)$ \\
\hline $\mathrm{C} 1 \mathrm{~B}-\mathrm{N} 1 \mathrm{~B}-\mathrm{H} 1 \mathrm{NB}$ & $125(2)$ \\
\hline $\mathrm{C} 7 \mathrm{~B}-\mathrm{N} 2 \mathrm{~B}-\mathrm{C} 6 \mathrm{~B}$ & $110.3(2)$ \\
\hline $\mathrm{C} 7 \mathrm{~B}-\mathrm{N} 2 \mathrm{~B}-\mathrm{H} 2 \mathrm{NB}$ & $121(2)$ \\
\hline $\mathrm{C} 6 \mathrm{~B}-\mathrm{N} 2 \mathrm{~B}-\mathrm{H} 2 \mathrm{NB}$ & $129(2)$ \\
\hline $\mathrm{C} 3 \mathrm{~B}-\mathrm{N} 3 \mathrm{~B}-\mathrm{H} 3 \mathrm{NC}$ & $116(2)$ \\
\hline $\mathrm{C} 3 \mathrm{~B}-\mathrm{N} 3 \mathrm{~B}-\mathrm{H} 3 \mathrm{ND}$ & $114(2)$ \\
\hline $\mathrm{H} 3 \mathrm{NC}-\mathrm{N} 3 \mathrm{~B}-\mathrm{H} 3 \mathrm{ND}$ & $111(3)$ \\
\hline $\mathrm{C} 2 \mathrm{~B}-\mathrm{C} 1 \mathrm{~B}-\mathrm{N} 1 \mathrm{~B}$ & $131.8(2)$ \\
\hline $\mathrm{C} 2 \mathrm{~B}-\mathrm{C} 1 \mathrm{~B}-\mathrm{C} 6 \mathrm{~B}$ & $122.1(2)$ \\
\hline $\mathrm{N} 1 \mathrm{~B}-\mathrm{C} 1 \mathrm{~B}-\mathrm{C} 6 \mathrm{~B}$ & $106.1(2)$ \\
\hline $\mathrm{C} 1 \mathrm{~B}-\mathrm{C} 2 \mathrm{~B}-\mathrm{C} 3 \mathrm{~B}$ & $117.3(2)$ \\
\hline $\mathrm{C} 1 \mathrm{~B}-\mathrm{C} 2 \mathrm{~B}-\mathrm{H} 2 \mathrm{~B}$ & $122.3(17)$ \\
\hline $\mathrm{C} 3 \mathrm{~B}-\mathrm{C} 2 \mathrm{~B}-\mathrm{H} 2 \mathrm{~B}$ & $120.4(17)$ \\
\hline $\mathrm{C} 2 \mathrm{~B}-\mathrm{C} 3 \mathrm{~B}-\mathrm{C} 4 \mathrm{~B}$ & $120.3(2)$ \\
\hline $\mathrm{C} 2 \mathrm{~B}-\mathrm{C} 3 \mathrm{~B}-\mathrm{N} 3 \mathrm{~B}$ & $119.0(2)$ \\
\hline $\mathrm{C} 4 \mathrm{~B}-\mathrm{C} 3 \mathrm{~B}-\mathrm{N} 3 \mathrm{~B}$ & $120.6(2)$ \\
\hline $\mathrm{C} 5 \mathrm{~B}-\mathrm{C} 4 \mathrm{~B}-\mathrm{C} 3 \mathrm{~B}$ & $122.0(3)$ \\
\hline $\mathrm{C} 5 \mathrm{~B}-\mathrm{C} 4 \mathrm{~B}-\mathrm{H} 4 \mathrm{~B}$ & $122.1(18)$ \\
\hline $\mathrm{C} 3 \mathrm{~B}-\mathrm{C} 4 \mathrm{~B}-\mathrm{H} 4 \mathrm{~B}$ & $115.9(18)$ \\
\hline $\mathrm{C} 4 \mathrm{~B}-\mathrm{C} 5 \mathrm{~B}-\mathrm{C} 6 \mathrm{~B}$ & $117.4(3)$ \\
\hline $\mathrm{C} 4 \mathrm{~B}-\mathrm{C} 5 \mathrm{~B}-\mathrm{H} 5 \mathrm{~B}$ & $124(2)$ \\
\hline $\mathrm{C} 6 \mathrm{~B}-\mathrm{C} 5 \mathrm{~B}-\mathrm{H} 5 \mathrm{~B}$ & $119(2)$ \\
\hline $\mathrm{C} 5 \mathrm{~B}-\mathrm{C} 6 \mathrm{~B}-\mathrm{N} 2 \mathrm{~B}$ & $132.9(2)$ \\
\hline $\mathrm{C} 5 \mathrm{~B}-\mathrm{C} 6 \mathrm{~B}-\mathrm{C} 1 \mathrm{~B}$ & $120.8(2)$ \\
\hline $\mathrm{N} 2 \mathrm{~B}-\mathrm{C} 6 \mathrm{~B}-\mathrm{C} 1 \mathrm{~B}$ & $106.3(2)$ \\
\hline $\mathrm{N} 2 \mathrm{~B}-\mathrm{C} 7 \mathrm{~B}-\mathrm{N} 1 \mathrm{~B}$ & $106.8(2)$ \\
\hline $\mathrm{N} 2 \mathrm{~B}-\mathrm{C} 7 \mathrm{~B}-\mathrm{S} 1 \mathrm{~B}$ & $126.69(19)$ \\
\hline $\mathrm{N} 1 \mathrm{~B}-\mathrm{C} 7 \mathrm{~B}-\mathrm{S} 1 \mathrm{~B}$ & $126.5(2)$ \\
\hline $\mathrm{C} 7 \mathrm{~B}-\mathrm{N} 1 \mathrm{~B}-\mathrm{C} 1 \mathrm{~B}-\mathrm{C} 2 \mathrm{~B}$ & $177.4(3)$ \\
\hline $\mathrm{C} 7 \mathrm{~B}-\mathrm{N} 1 \mathrm{~B}-\mathrm{C} 1 \mathrm{~B}-\mathrm{C} 6 \mathrm{~B}$ & $-1.7(3)$ \\
\hline $\mathrm{N} 1 \mathrm{~B}-\mathrm{C} 1 \mathrm{~B}-\mathrm{C} 2 \mathrm{~B}-\mathrm{C} 3 \mathrm{~B}$ & $-177.8(2)$ \\
\hline $\mathrm{C} 6 \mathrm{~B}-\mathrm{C} 1 \mathrm{~B}-\mathrm{C} 2 \mathrm{~B}-\mathrm{C} 3 \mathrm{~B}$ & $1.2(4)$ \\
\hline $\mathrm{C} 1 \mathrm{~B}-\mathrm{C} 2 \mathrm{~B}-\mathrm{C} 3 \mathrm{~B}-\mathrm{C} 4 \mathrm{~B}$ & $-2.9(4)$ \\
\hline $\mathrm{C} 1 \mathrm{~B}-\mathrm{C} 2 \mathrm{~B}-\mathrm{C} 3 \mathrm{~B}-\mathrm{N} 3 \mathrm{~B}$ & $-179.0(2)$ \\
\hline $\mathrm{C} 2 \mathrm{~B}-\mathrm{C} 3 \mathrm{~B}-\mathrm{C} 4 \mathrm{~B}-\mathrm{C} 5 \mathrm{~B}$ & $2.4(4)$ \\
\hline $\mathrm{N} 3 \mathrm{~B}-\mathrm{C} 3 \mathrm{~B}-\mathrm{C} 4 \mathrm{~B}-\mathrm{C} 5 \mathrm{~B}$ & $178.5(3)$ \\
\hline $\mathrm{C} 3 \mathrm{~B}-\mathrm{C} 4 \mathrm{~B}-\mathrm{C} 5 \mathrm{~B}-\mathrm{C} 6 \mathrm{~B}$ & $0.0(4)$ \\
\hline $\mathrm{C} 4 \mathrm{~B}-\mathrm{C} 5 \mathrm{~B}-\mathrm{C} 6 \mathrm{~B}-\mathrm{N} 2 \mathrm{~B}$ & $177.3(3)$ \\
\hline $\mathrm{C} 4 \mathrm{~B}-\mathrm{C} 5 \mathrm{~B}-\mathrm{C} 6 \mathrm{~B}-\mathrm{C} 1 \mathrm{~B}$ & $-1.8(4)$ \\
\hline $\mathrm{C} 7 \mathrm{~B}-\mathrm{N} 2 \mathrm{~B}-\mathrm{C} 6 \mathrm{~B}-\mathrm{C} 5 \mathrm{~B}$ & $-179.4(3)$ \\
\hline $\mathrm{C} 7 \mathrm{~B}-\mathrm{N} 2 \mathrm{~B}-\mathrm{C} 6 \mathrm{~B}-\mathrm{C} 1 \mathrm{~B}$ & $-0.3(3)$ \\
\hline $\mathrm{C} 2 \mathrm{~B}-\mathrm{C} 1 \mathrm{~B}-\mathrm{C} 6 \mathrm{~B}-\mathrm{C} 5 \mathrm{~B}$ & $1.2(4)$ \\
\hline $\mathrm{N} 1 \mathrm{~B}-\mathrm{C} 1 \mathrm{~B}-\mathrm{C} 6 \mathrm{~B}-\mathrm{C} 5 \mathrm{~B}$ & $-179.6(2)$ \\
\hline $\mathrm{C} 2 \mathrm{~B}-\mathrm{C} 1 \mathrm{~B}-\mathrm{C} 6 \mathrm{~B}-\mathrm{N} 2 \mathrm{~B}$ & $-178.1(2)$ \\
\hline $\mathrm{N} 1 \mathrm{~B}-\mathrm{C} 1 \mathrm{~B}-\mathrm{C} 6 \mathrm{~B}-\mathrm{N} 2 \mathrm{~B}$ & $1.2(3)$ \\
\hline
\end{tabular}

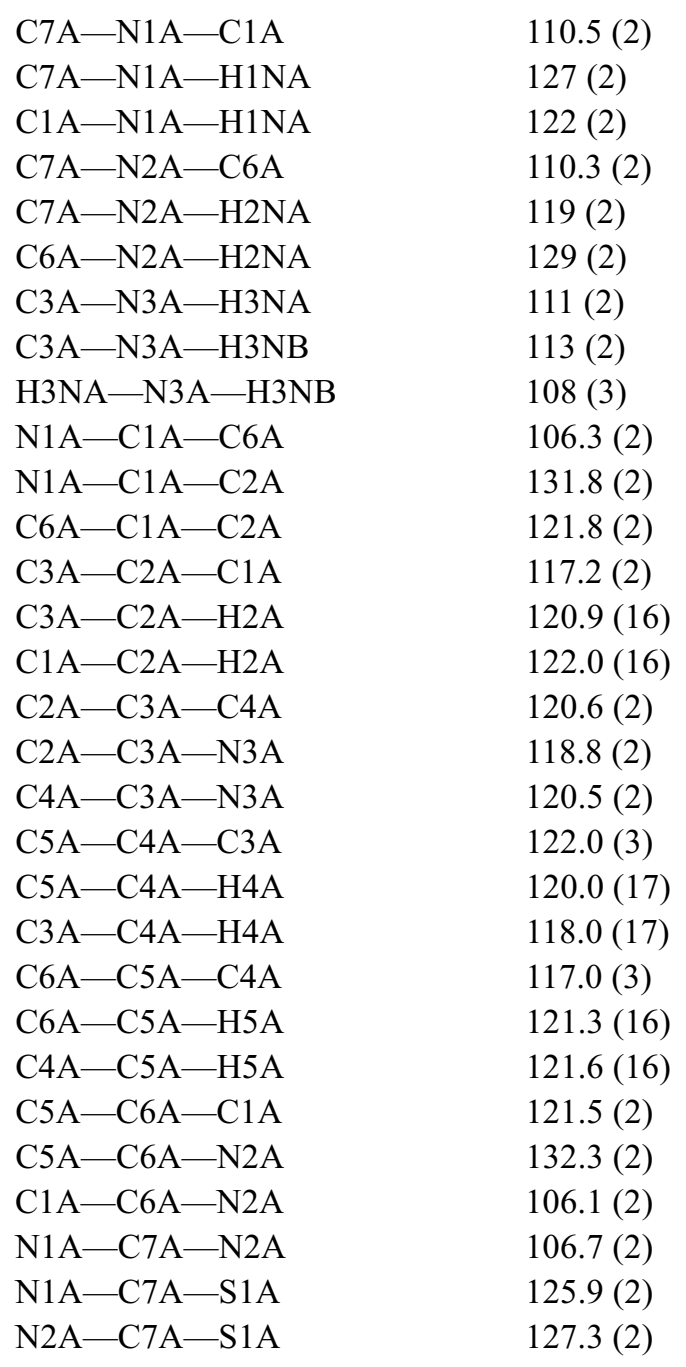

110.5 (2)

127 (2)

$122(2)$

$110.3(2)$

119 (2)

$129(2)$

$111(2)$

$113(2)$

$108(3)$

$106.3(2)$

$131.8(2)$

$121.8(2)$

$117.2(2)$

120.9 (16)

$122.0(16)$

$120.6(2)$

$118.8(2)$

$120.5(2)$

$122.0(3)$

$120.0(17)$

$118.0(17)$

$117.0(3)$

$121.3(16)$

$121.6(16)$

$121.5(2)$

$132.3(2)$

$106.1(2)$

106.7 (2)

$125.9(2)$

$127.3(2)$

$1.4(3)$

$-175.1(3)$

$174.9(3)$

$-1.1(4)$

$1.8(4)$

$178.6(2)$

$-0.8(4)$

$-177.6(2)$

$-0.8(4)$

$1.5(4)$

$-174.7(3)$

$-177.5(2)$

$-0.6(4)$

$-0.4(3)$

$176.5(2)$

$176.0(3)$

-0.7 (3) 


$\begin{array}{llll}\mathrm{C} 6 \mathrm{~B}-\mathrm{N} 2 \mathrm{~B}-\mathrm{C} 7 \mathrm{~B}-\mathrm{N} 1 \mathrm{~B} & -0.8(3) & \mathrm{C} 1 \mathrm{~A}-\mathrm{N} 1 \mathrm{~A}-\mathrm{C} 7 \mathrm{~A}-\mathrm{N} 2 \mathrm{~A} & -1.8(3) \\ \mathrm{C} 6 \mathrm{~B}-\mathrm{N} 2 \mathrm{~B}-\mathrm{C} 7 \mathrm{~B}-\mathrm{S} 1 \mathrm{~B} & 179.5(2) & \mathrm{C} 1 \mathrm{~A}-\mathrm{N} 1 \mathrm{~A}-\mathrm{C} 7 \mathrm{~A}-\mathrm{S} 1 \mathrm{~A} & 177.73(19) \\ \mathrm{C} 1 \mathrm{~B}-\mathrm{N} 1 \mathrm{~B}-\mathrm{C} 7 \mathrm{~B}-\mathrm{N} 2 \mathrm{~B} & 1.5(3) & \mathrm{C} 6 \mathrm{~A}-\mathrm{N} 2 \mathrm{~A}-\mathrm{C} 7 \mathrm{~A}-\mathrm{N} 1 \mathrm{~A} & 1.6(3) \\ \mathrm{C} 1 \mathrm{~B}-\mathrm{N} 1 \mathrm{~B}-\mathrm{C} 7 \mathrm{~B}-\mathrm{S} 1 \mathrm{~B} & -178.68(19) & \mathrm{C} 6 \mathrm{~A}-\mathrm{N} 2 \mathrm{~A}-\mathrm{C} 7 \mathrm{~A}-\mathrm{S} 1 \mathrm{~A} & -177.99(19)\end{array}$

Hydrogen-bond geometry $\left(A,{ }^{\circ}\right)$

\begin{tabular}{lllll}
\hline$D-\mathrm{H} \cdots A$ & $D-\mathrm{H}$ & $\mathrm{H} \cdots A$ & $D \cdots A$ & $D-\mathrm{H} \cdots A$ \\
\hline $\mathrm{N} 1 A-\mathrm{H} 1 N A \cdots \mathrm{N} 3 B$ & $0.80(3)$ & $2.06(3)$ & $2.856(3)$ & $176(3)$ \\
$\mathrm{N} 2 A-\mathrm{H} 2 N A \cdots \mathrm{S} 1 B^{\mathrm{i}}$ & $0.82(3)$ & $2.54(3)$ & $3.295(2)$ & $154(3)$ \\
$\mathrm{N} 3 A-\mathrm{H} 3 N A \cdots \mathrm{S} 1 A^{\text {ii }}$ & $0.84(4)$ & $2.75(4)$ & $3.551(3)$ & $159(3)$ \\
$\mathrm{N} 3 A-\mathrm{H} 3 N B \cdots \mathrm{C} 4 B^{\mathrm{iii}}$ & $0.89(4)$ & $2.71(4)$ & $3.483(4)$ & $145(3)$ \\
$\mathrm{N} 3 A-\mathrm{H} 3 N B \cdots \mathrm{C} 5 B^{\mathrm{iii}}$ & $0.89(4)$ & $2.81(4)$ & $3.643(4)$ & $155(3)$ \\
$\mathrm{N} 1 B-\mathrm{H} 1 N B \cdots \mathrm{N} 3 A^{\text {iv }}$ & $0.88(3)$ & $2.02(3)$ & $2.884(3)$ & $171(3)$ \\
$\mathrm{N} 2 B-\mathrm{H} 2 N B \cdots \mathrm{S} 1 A^{\mathrm{v}}$ & $0.85(3)$ & $2.56(3)$ & $3.340(2)$ & $153(3)$ \\
$\mathrm{N} 3 B-\mathrm{H} 3 N C \cdots \mathrm{S} 1 B^{\mathrm{vi}}$ & $0.86(3)$ & $2.91(3)$ & $3.672(3)$ & $149(2)$ \\
$\mathrm{N} 3 B-\mathrm{H} 3 N D \cdots \mathrm{S} 1 B^{\mathrm{vii}}$ & $0.85(3)$ & $2.70(3)$ & $3.477(3)$ & $153(3)$ \\
$\mathrm{C} 5 A-\mathrm{H} 5 A \cdots \mathrm{S} 1 A^{\text {viii }}$ & $0.96(3)$ & $2.96(3)$ & $3.656(3)$ & $130.2(19)$ \\
$\mathrm{C} 5 B-\mathrm{H} 5 B \cdots \mathrm{C} 1 A^{\text {ii }}$ & $0.90(3)$ & $2.78(3)$ & $3.562(4)$ & $147(3)$
\end{tabular}

Symmetry codes: (i) $-x+1, y+1,-z+1 / 2$; (ii) $-x+3 / 2, y-1 / 2,-z+1 / 2$; (iii) $x,-y+1, z+1 / 2$; (iv) $-x+1, y,-z+1 / 2$; (v) $x, y-1, z$; (vi) $-x+1,-y+1,-z$; (vii) $x+1 / 2, y+1 / 2, z$; (viii) $x,-y+2, z+1 / 2$. 